\title{
TRANSPLANTATION PSYCHIATRY: AN OVERVIEW
}

\author{
Vesna Medved $^{1,2}$, Sara Medved $^{1}$ \& Milena Skočić Hanžek ${ }^{1,2}$ \\ ${ }^{1}$ Department of Psychiatry, University Hospital Centre Zagreb, Zagreb, Croatia \\ ${ }^{2}$ School of Medicine, University of Zagreb, Zagreb, Croatia
}

received: 20.2.2018;

revised: 21.6.2018;

accepted: 10.7 .2018

\section{SUMMARY}

Psychiatric consultation and evaluation is an important part of candidate selection for organ transplantation. Psychiatric assessment of patients undergoing transplantation procedure is done in pre-and post-transplantation periods, each one having its specifics. In the pre-transplantation period it is crucial to assess the patient's therapeutic adherence and the ability of understanding the treatment. The main prediction factor for short and long-term success is medical compliance for which thorough clarification of psychosocial support is needed. Symptoms of psychological exhaustion due to physical illness should be distinguished from psychiatric disorders. If a patient has a history of psychiatric illness, the risks of exacerbation or recurrence of a disease need to be evaluated. Pharmacokinetic and pharmacodynamics of psychotropic drugs resulting from to organ failure should be considered when deciding on treatment. Psychiatric assessment of the organ donor aims to clarify the psychological suitability and motivation. There are specific aspects of liver, kidney, heart and lung transplantation to keep in mind. Understanding psychosexual development is particularly important to children, before transplantation, as is the assessment of every family member. The consultation concludes with an overall evaluation of the patient's psychosocial strengths and limitations and recommended interventions to optimize the candidacy for transplantation. In the post-transplantation period potential psychological problems or psychiatric disorders must be identified and treated accordingly, in addition to psychiatric side effects of immunosuppressive therapy. The use of psychotropic drugs in the post-transplantation period requires knowledge of medication interactions.

Overall, psychiatrists perform multiple roles in the transplantation team. The psychiatrists' goals are to meet the psychological needs of both patients and potential donors, evaluate candidates and to help other medical experts on the team with understanding underlying psychological mechanisms triggered by serious medical conditions and procedures. Finally, the most important purpose is optimal organ recruitment and recovery.

Key words: transplantation - psychiatry - psychotropic drugs - immunosuppressive agents

$* * * * *$

\section{INTRODUCTION}

Despite dramatic improvements in surgical techniques and immunosuppressive drugs, the number of transplants falls short of the need. Significant increase in transplantation can be seen in almost all solid ograns transplantation: kidney, liver, heart, lung and pancreas.

To optimize recovery and outcomes, candidates for transplantation process are, among other factors, selected by psychiatric and psychosocial factors. Psychiatry in transplantation medicine is a sub specific area; it represents a psychiatric-ethical and legal judgment in different phases of the transplantation process (Anil Kumar \& Mattoo 2015).

Worldwide, transplantation centers have different protocols for psychiatric evaluation of patients in the transplantation process. Some first apply screening questionnaires as General Health Questionnaire, and after continue to psychiatric evaluation. Others apply a range of self-scored instruments for depression, anxiety or delirium, afterwards continuing with interviewing. Most commonly the evaluation begins with non-structured psychiatric clinical interview, followed by the application of scoring instruments, neuropsychological testing and social service evaluations if needed.

Psychiatric interviews are required to assess the patients' ability to provide informed consent and to identify potential psychological problems or psychiatric disorders that could disrupt the transplantation process. There are three key elements in assessing a patient's ability to provide informed consent: adequate information about the process of transplantation and perceived ability to understand and make independent decisions. Since the waiting period between getting on a list until transplantation can take up to several years, psychiatric evaluations can be carried out several times. It was shown that psychiatric evaluation in pre-transplantation period can predict post-transplant psychiatric adjustment (Dew et al. 2000).

\section{PSYCHIATRIC ASPECTS OF PRE-TRANSPLANTATION PERIOD}

Deterioration in somatic health inevitably leads to complex behavioral reactions and changes in emotional functioning. Furthermore, psychological reactions to illness affect the relationship and communication with medical staff and can change the therapy adherence. The psychological response depends on the nature of the disease itself, personality of the patient, maturity of the patient's personality structure and does not necessarily have to be characterized as psychopathological. Phenomena that can occur as a reaction to a serious illness are: negation or underestimation of illness, anger, depres- 
sion, isolation. In terminal patients awaiting transplantation, these phenomena often appear rather chaotic and can be difficult to treat.

Getting on the transplantation list is seen as a chance for a new life and is often followed by elevated moods close to euphoria. However, a prolonged waiting period, in which contact with transplantation team is scarce, may cause patients to develop feelings of resentment; they may feel forgotten and abandoned. Accumulated frustrations can thus cause acting out to medical personnel, who in turn reactively avoid such patients, creating a vicious cycle of difficult relationships.

Studies indicate show that 4.5 to 64 percent of patients awaiting transplants suffer from depression (a wide variance is ascribed to different diagnostic methods used (Bianchi et al. 2005)). Studies have found presence of anxiety disorders, mixed anxiety and depression, and adjustment disorders in 36 percent of patients in the pretransplantation period (Rogal et al. 2011). Some patients are, due to their poor somatic state, forced to wait for the transplants in intensive care units. During this period, a family-like relationship between the patient and the medical staff is likely to develop. It is not uncommon for patients awaiting transplants to become competitive about potential operation schedules. Feelings of envy, suspicion, aggression, instability emerge as some patients get much needed organs sooner than others. In such situations a psychiatrist intervention is required to calm patients through psychotherapeutic and, if needed, psychopharmacological treatment. Psychiatrists also help other members of the medical staff to understand difficult psychological reactions that might otherwise be seen as patient non-compliance, or even a psychiatric disorder. Misunderstandings in communication and the physicians' expectations can have implications for diagnostic and therapeutic procedures. With help, mutual understanding leads to improved satisfaction even when the expected physical improvement in treatment is absent.

In pre-transplantation periods a psychiatrist should distinguish symptoms of psychological exhaustion due to physical illness or from clinical depression. Psychological exhaustion manifests as lack of will, fatigue, disorders of attention and is a normal response in a context of serious medical illness. Psychiatric changes may also be iatrogenic. For example, depression is a frequent side effect of interferon treatment for hepatitis C. In such cases an initial psychiatric evaluation would spot iatrogenic depression.

Problems in a patient's emotional, social or financial life can lead to a downward psychological spiral. Therefore, psychosocial evaluation is required and involves identifying persons willing to provide the patient with the necessary help in daily activities (supervising the consumption of prescribed therapy, regular medical monitoring, etc.). Patient's financial situation, housing and hygiene conditions should be considered and, if necessary, intervention by the amenable social welfare center should be requested. If some of the criteria are not satisfactory, the process of making the right to financial aid or the process of accommodation in temporary foster care can be organized.

Thus, the evaluation of post-transplant compliance is of outmost importance in transplantation psychiatry. A thorough psychological evaluation can help identify methods for improving a patient's long-term therapeutic compliance. Long term compliance is the greatest predictor of success.

\section{Contraindications for transplantation}

As in any medical procedure, there are absolute and relative contraindications (Table 1). Any patient who has a known history of therapeutic noncompliance will probably present that same behavior after transplantation. Compliance refers to the continuous taking of prescribed immunosuppressive therapy, but also to general compliance in treatment (regular checkups, alcohol abstinence, physical activity, etc.). Both types of therapeutic noncompliance range from 15 percent to 40 percent (Burra et al. 2011). Hence, compliance is the main parameter when approving suitability for transplantation candidacy. Patients prone to therapeutic noncompliance usually have one or more of the following risk factors: psychiatric disorder, younger than 40, side effects of immunosuppressive therapy and a belief that the drugs are poisonous (Rovelli et al. 1989). Accordingly, psychiatric assessment needs to identify candidates who are more likely to be compliant with therapeutic regimes and preserve the transplanted organ.

Table 1. Psychiatric contraindications for transplantation

\begin{tabular}{ll}
\hline Absolute & Relative \\
\hline $\begin{array}{l}\text { Dementia } \\
\text { Acute psychosis }\end{array}$ & Therapeutic noncompliance \\
$\begin{array}{l}\text { Current physical } \\
\text { addiction to drugs } \\
\text { and/or alcohol }\end{array}$ & Earlier psychiatric disorders \\
$\begin{array}{l}\text { Extremely unstable } \\
\text { borderline }\end{array}$ & Depression \\
IQ less than 70 & $\begin{array}{l}\text { Anxiety disorders } \\
\end{array}$ \\
& $\begin{array}{l}\text { Limited family/social support } \\
\text { Demotivation for the procedure } \\
\text { Contraindications for transplantation }\end{array}$ \\
\hline
\end{tabular}

As the most common cause for liver transplantation is alcoholism, compliance requires complete abstinence from alcohol. Almost all transplant centers require at least 6 months of abstinence. Longer abstinence lowers the risk of alcoholism recurrence after transplantation (Tandon et al. 2009). Alcohol consumption after transplantation due to alcohol liver cirrhosis is seen as noncompliance (Adam et al. 2003). In addition to the length of pre-transplantation abstinence, post-transplantation predictors of alcoholism recurrence are: personality disorders, hereditary burden of alcoholism and polytoxicomania. It is estimated that five years after liver transplantation up to 50 percent of patients will relapse, that 
is start drinking alcohol (Mackie et al. 2001, Tome \& Lucey 2003). Factors that are related to alcohol abstinence are: older age, social security, therapeutic compatibility, and absence of co-morbid psychiatric disorders (McCallum \& Masterton 2006).

The second most common reason for liver transplantation is hepatitis $\mathrm{C}$ in intravenous drug addicts. During abstinence from intravenous drug consumption (mostly opioids), substitution therapy is necessary. In preparation for transplantation, some psychiatrists gradually reduce maintenance therapy, which in up to 80 percent of patients leads to opioid addiction recurrence and, consequently, automatic removal from the transplant list (Robaeys et al. 2009). For this reason it is advised that dosage of substitution therapy is carefully monitored both in the pre- and post-transplant period. Moreover, strong psychosocial support must be provided.

Antisocial personality disorder in comorbidity with polytoxicomania is a powerful predictor of post-transplant alcohol and drug consumption and the most common psychiatric reason for restricting placement on the list (Bunzel \& Laederach-Hofmann 2000). Patients with anti-social personality disorder are twice as much more likely to relapse in terms of alcohol and/or narcotic addiction in comparison to patients without this particular personality disorder (Coffman et al. 1997). Diagnosis of extremely unstable borderline personality combined with history of suicide attempts is an absolute contraindication for listing (Yates et al. 1998).

In terms of intellectual disabilities, 70 percent of the transplantation centers in the world believe that the intelligence quotient score less than 70 is an absolute contraindication for transplantation, but in scientific community and clinical practice there is an ongoing debate on whether such an attitude is a form of discrimination (Panocchi et al. 2010).

Mild cognitive impairment decreases a patient's ability to learn new requirements regarding drugs, medical assessment, diet etc. Accordingly, in those patients it is vital to estimate the support of the potential caregiver. When assessing cognitive status, it is important to distinguish potentially reversible somatic condition (hepatic encephalopathy, uremic encephalopathy) from previously existing dementia. Encephalopathy is an indication for urgent transplantation, while dementia is a contraindication (Teperman 2013).

Patients who develop either severe depressive symptoms or suffer from intense anxiety are not eligible for listing because of potentially poor therapeutic compliance or even self-destructive and suicidal behavior. It is necessary to conduct psychiatric treatment and establish a stable remission after which they can be placed on the transplantation list. In these patients, drug dosage in pre- and post-transplant period remains the same. Sometimes there is a dilemma whether psychotic disorder is a contraindication for listing. Each patient needs to be individually evaluated. Acute psychosis is an absolute contraindication, while a psychotic patient who is in continuous treatment, in a stable remission, with good therapeutic compliance and reliable social support can be placed on the list. According to our clinical experience, as well as the literature quotations, such transplants are in most cases successful (Bouhlel 2014). However, in about a quarter of stable schizophrenic patients there is a possibility of postoperative relapse of psychosis. The risk is even greater for those undergoing bone marrow transplantation after which they must be held in isolation for a long time period of time. Therefore, daily psychiatric monitoring of transplanted schizophrenic patients is needed in order to ensure safety both of the patient and the staff (Akaho et al. 2003).

There are situations when an urgent transplantation is required and consequently urgent psychiatric assessment is needed. Such situations can be caused by failed suicide attempts; ergo it is necessary to determine whether an isolated attempt of suicide is precipitated by external factors or recurrent as a result of psychiatric disorder. In cases where it is impossible to talk to the patient directly due to their physical state, the assessment is based on the data obtained from the family or close friends.

\section{Psychiatric evaluation of the donor}

The decision to donate an organ is a difficult one. The donor is most often a family member who is emotionally related to the patient and has volunteered. In psychiatric assessments we are always led to make decisions in the best interest of the donor. Psychiatric interviews are initially conducted separately with the donors and the recipients, and later jointly. The purpose of the evaluation is to determine several crucial points. First, is the donor able to provide an informed consent and is the donor aware of possible post-operative risks. Psychiatrists need to explore the impact of the donation: will it have an effect on the donor's health, will it decrease his ability to work, etc. And lastly, psychiatrists need to investigate the motivation for the donation and the nature of the relationship with the recipient.

Based on our clinical experience, we estimate that only about half of the donors (liver segment) are socalled "unconditional donors", people who would emotionally suffer from being disallowed of the donation. The second category consists of impulsive donations, family pressured donations, people who expect compensation and those who lack information about the process. All of these motives for organ donations are medically unacceptable. Altruistic (unrestrained) donation means that the potential donor is motivated solely by care for the well-being of another person. It should be distinguished from feelings of loyalty and moral commitments that bind potential donors to a particular potential organ recipient. In the case of an altruistic donation the donor does not know who the recipient is and vice versa. Reward for donation is the donor's own sense of gratification. It is not easy to recognize pure altruism as motivation and to uncover possible material 
(financial) gain of potential donors. Therefore, our attitude, but also the practice of other centers, is to avoid unrelated donations (Carney 2017).

Donors benefit from consulting with psychiatrist mostly because it enables them to become fully aware of their reasons for the procedure. Unconscious feelings toward the recipient of the organ can be explored, as well as any unrealistic expectations of the recipient. Donor's withdrawal may cause negative reactions in a potential recipient and its' environment. Such donor needs to be presented to the family as an unacceptable donor for medical reasons, whatever the reason for the withdrawal is.

\section{PSYCHIATRIC ASPECTS OF THE POST-TRANSPLANT PERIOD}

During extended convalescence, patients often develop comorbidities and complications. Infections, diabetes, hypertension, cardiovascular disease, osteoporosis and renal failure are among common consequences of immunosuppressive therapy, as are acute and chronic rejections of grafts. These conditions are accompanied by the patient's continuous fear of organ rejection, fear of the development of new comorbidities and fear of death (Schulz \& Kroencke 2012).

In a small number of patients, transplantation could induce post-traumatic psychological maturation in different personality domains. This manifests itself as increasing sociability, as well as feeling stronger or more creative. It is also expressed through the feelings of having a second chance to re-create life (Zoellner \& Maercker 2006). However, there are several possible mental disorders that can occur in the post-transplant period. Some patients can have problems with psychological integration of graft and develop a distorted body image.

However, the most prevalent post-transplant psychiatric problem is delirium. It develops over several hours or days, manifesting itself as an agitated condition with hallucinations or as a state of somnolence. If there are no neurological signs it is difficult to clinically distinguish it from the neurotoxic effect of tacrolimus. Magnetic resonance images of tacrolimus leukoencephalopathy caused by its' neurotoxic effect is depicted as a white brain edema in the back regions of the brain (Mi et al. 2017). To our best knowledge, there is still no effective prevention strategy for delirium (Inouye et al. 2013). Symptomatic therapy for agitated delirium is haloperidol or one of atypical antipsychotics. Studies regarding use of psychotropic medicine in delirium treatment are scarce and only one reported beneficial effect of quetiapine. When compared to other antipsychotics, quetiapine has shortened the duration of delirium and reduced agitation (Gilchrist et al. 2012). Antipsychotics are regularly used to relieve delirium, which can last for several weeks.
Therapeutic co-morbidity of patients is of crucial importance for the preservation of grafts. Studies report that 23 percent of transplanted patients are not taking immunosuppressive therapy as prescribed and up to 50 percent of acute rejection results from the termination of drug use. Therapeutic instability happens more often in adolescents and in patients with transplanted kidneys (De Bleser et al. 2009, Dew et al. 2007).

Psychiatric disturbances may occur in donors as well, in the sense of adjustment disorders or hypochondria. The rejection of the donated organ can cause the donor to feel guilty, anxious, and depressed. The same reaction may develop when transplantation is successful, but postoperative complications in the donor appear (Parikh et al. 2010).

\section{PSYCHIATRIC ASPECTS OF LIVER TRANSPLANTATION}

The need for liver transplantation exceeds the number of transplantations performed in a year, despite the possibility of living donors (Hart et al. 2017). About 90 percent of liver transplantation is done because of alcohol cirrhosis. For that reason, abstinence establishment is crucial. In consequence, the main scope of preoperative psychiatric examination in patients with alcoholism is the assessment of therapeutic cooperation and risk of recurrence. Since recurrence of alcoholism in transplanted alcoholic liver disease can be up to 50 percent (Iruzubieta et al. 2013), good psychosocial support needs to be established. Patients with alcoholic liver cirrhosis who do not have problems in family interactions and socioeconomic consequences of drinking are more likely to maintain abstinence.

\section{PSYCHIATRIC ASPECTS OF RENAL TRANSPLANTATION}

The most frequent psychological problems in patients awaiting renal transplantation are depression and anxiety, often combined with poor compliance regarding diet and medications (Naqui 2015). Since renal donation from living donors is very common, psychiatrist must estimate the donor's knowledge about the transplantation process and whether the donation is voluntary. As in other living donations, it may be an impulse decision triggered by emotions, material compensation or by family pressure. If a donor is a member of family, important factor is family dynamics; the strength of the bonds between family members, the position of the donor or the recipient and the decision-making member(s) of the family. Renal recipients with failing graft can sense the guilt towards the donor, because of organ waste. Also, they face the fear of not having the support for another transplant within the family. In that case, the psychiatrist is supposed to provide psychological support and counselling to the patient in order to reduce emotional tension and other possible psychological reactions. 


\section{PSYCHIATRIC ASPECTS OF LUNG TRANSPLANTATION}

The scope of psychiatric evaluations of patients who are candidates for lung transplantation is not just diagnosing conditions that are contraindications for transplantation but also recognizing the state of psychological stress in these patients. Their lungs are in a prolonged state of functional decline, which leads to restriction in physical activity, complex medical regimens, and the necessity for nursing care. More than 47 percent of patients awaiting lung transplantation have anxiety disorder or adjustment disorders, with 28 percent diagnosed with either panic or generalized anxiety disorder (Parekh et al. 2003). Moreover, incidence of anxiety disorders is far greater than in the general population and is associated with higher mortality after transplantation (Smith et al. 2016). Because post-transplantation monitoring and treatment regimen is complex and requires continuous support by a caregiver, lack of sufficient care provider represents a potential psychosocial contraindication for lung transplantation (Barbour et al. 2006).

\section{PSYCHIATRIC ASPECTS OF HEART TRANSPLANTATION}

From a psychological perspective, the heart is an organ that has a symbolic nature as a center of one's personality, including all memories, thoughts, desires, fantasies and feelings. Studies have shown that even when recipients possess the knowledge of the anatomy and physiology, they still express fantasies and magical thinking regarding loss of the old heart and acquisition of the new heart (Inspector et al. 2004). However, a new study finds that patients with heart transplantation integrated the new organs well, with a subgroup of 1520 percent of patients who had body image problems (Hartmann et al. 2017). Among inpatients waiting for heart transplantation, anxiety symptoms are more prevalent (around 47 percent) than depressive (around 2 percent prevalence) (Chenyu et al. 2013). Living after a heart transplant is complex: recipients are confronted with lifelong immunosuppressive therapy associated with many side-effects and a constant fear of physiological rejection of the new heart and finally death (Sadala \& Stolf 2008). The prevalence of depression is common among heart transplant recipients especially among patients with comorbidities (Milaniak et al. 2017). Mechanisms such as acceptance/optimism, setting targets, making attributions, seeking social support, having faith and changing priorities and perceptions are related to better life quality (Kaba et al. 2005).

Simultaneous transplantation of heart and lungs represent certainly a moment to rejoice and appreciate being given a new lease on life and a clear sense of new priorities (in particular regarding interpersonal relationships and a shift to altruism and spirituality) (Vamos 2010).

\section{PSYCHIATRIC ASPECTS OF TRANSPLANTATION IN CHILDHOOD}

The most important factor affecting a child's ability to cope with treatment is the response of her parents to the disease and treatment. Parents can however express feelings such as guilt or fear through disorganized, agitated, uncontrolled or demanding behavior that causes problems in communication with medical staff, as well as with the child. Their behavior must be understood as a high level anxiety reaction to having a seriously ill child. Family dynamics, communication and behavioral patterns must be evaluated and addressed. The most common pathological features are chaotic and unstable family relations. Alcohol and/or drug abuse, serious financial problems, verbal and even physical violence are extreme examples of chaotic families. In an unstable family constellation, the parental role can be spread among other family members, so it is not uncommon that grandparents take care of the ill child. If that is the case, grandmothers or grandfathers must be evaluated as primary caregivers and/or as potential donors.

Disorganized families are in risk for noncompliance, that is, for nonregulated medication of a child organ recipient. Falkenstein reported graft loss in up to 50 percent of noncompliance cases (Falkenstein et al. 2004). Inappropriate tacrolimus concentration in children after liver transplantation is a greater cause of loss of grafts than acute rejection. Thus, blood levels of tacrolimus must be regularly monitored. Psychiatric interventions are targeted at parental dysfunction, communication difficulties and provision of support during the treatment. Direct confrontation regarding regular drug use is not helpful as primary intervention.

Parents are under constant psychological strain. At first from the burden of chronic illness awaiting transplantation to post-transplantation period, which sometimes takes up to ten years after the transplant. Up to one third of parents have some of the symptoms of posttraumatic stress disorder: avoiding behavior, impulsive thoughts or irritability (Muscara et al. 2015).

Children react to chronic illness through the defense mechanism of regression, which manifests as a fear and isolation or aggression and anger. Such psychological states make them less competent in social, physical and educational activities (Felnhofer et al. 2016). Children whose regressive behavior assumes having more confidence and faith in adults are more cooperative, while those who deny the disease are more aggressive, rebellious and unreliable in caring for themselves.

\section{PSYCHIATRIC SIDE EFFECTS OF IMMUNOSUPPRESSIVE THERAPY}

Immunosuppressive drugs that have to be taken for a lifetime have many psychological and neurological side effects. 
Cyclosporine can cause anxiety, delirium, hallucinations, epileptic attacks, tremor, paresthesia or cerebellar blindness. From our clinical experience, physicians in intensive care units often recognize somatic side effects of cyclosporine, however the problem arises when manifestations are exclusively psychiatric (i.e. delirium). Magnetic resonance scan of the brain must be made immediately, as it shows typical changes and confirms diagnosis. Simultaneous consumption of cyclosporine and lithium leads to increased lithium blood levels via increased tubular reabsorption. Tacrolimus use is linked to anxiety, restlessness, insomnia and delirium. Mycophenolate mofetil can incite anxiety, depression and somnolence. Corticosteroids induce a wide range of psychological side effects among which delirium, euphoria, depression, insomnia and irritability are most common (Taylor et al. 2010).

\section{THE USE OF PSYCHOPHARMACOLOGICAL DRUGS IN PRE- AND POST-TRANSPLANT PERIOD}

Depression is the most common psychiatric disorder in patients undergoing transplantation process (Jowsey et al. 2001). Selecting antidepressants in pre-transplantation phases requires special consideration upon changes in pharmacokinetics and interactions after transplantation. Also, dosage should be one-third to one-half of the usual therapeutic doses. A good profile of side effects makes selective serotonin re-uptake inhibitors (SSRIs) antidepressants of first choice. However, some of them, via inhibitory action of the CYP3A4 enzyme, may increase concentration of immunosuppressive drugs, leading to toxic side effects. The most potent inhibitor is fluvoxamine, and some inhibitory properties have fluoxetine, paroxetine and sertraline, while citalopram has no interaction with that enzyme (Taylor et al. 2010). Cyclosporine may increase blood concentration of lithium, fluoxetine and fluvoxamine. Venlafaxine may have cardiovascular side effects due to its norepinephrine effect, among which hypertension and tachycardia present a problem in patients who already have cardiovascular side effects of immunosuppressive therapy (Khan et al. 1991). Bupropion may lower the epileptogenic threshold. As immunosuppressive drugs are neurotoxic, bupropion should be used with caution (Taylor et al. 2010).

In transplanted patients with dyslipidemia and/or increased body weight, the use of mirtazapine may further aggravate the above mentioned conditions. Mirtazapine use is therefore justified in cachectic patients (Montgomery 1995). Tricyclic antidepressants (TCA-s) due to their significant anticholinergic and cardiovascular side effects (prolongation of QRS complex, atrial fibrillation, atrioventricular block, ventricular tachycardia) are contraindicated for patients after heart transplantation. Monoamine oxidase (MAO) inhibitors can cause hypertensive crisis. With SSRIs, MAO inhibitors can cause malignant hyperthermia and renal failure so their com- bined use is contraindicated. Patients should be warned of the potential risk of various herbal remedies because of interactions that lead to reduction of tacrolimus and cyclosporine blood concentrations of up to 50 percent (Mai et al. 2003).

Regarding anxiolytics, lorazepam, oxazepam and temazepam should be used due to their short-term elimination and the absence of active metabolites. As previously mentioned, patients waiting for or having had lung transplantation, are at greater risk of having one or more comorbid anxiety disorders. In this subgroup, quetiapine is a drug of choice, while benzodiazepines should be avoided due to their effect on respiratory suppression (Taylor et al. 2010).

If lithium is to be used in patients on dialysis, a single dose of $600 \mathrm{mg}$ daily is recommended after each dialysis session. In end stage renal disease dose reduction is generally not necessary, except for midazolam and chlordiazepoxide whose active metabolites are excreted through kidney (Taylor et al. 2010).

In the case of psychotic disorder, the use of haloperidol or quetiapine is advised due to its short half-life of elimination. In psychotic patients undergoing liver transplantation, it should be kept in mind that all antipsychotics are metabolized via liver enzymes except for sulpiride, amisulpride and paliperidone.

With immunosuppressive therapy, it is advised to avoid antipsychotic therapy in the form of long-lasting intramuscular injections.

Data regarding benefits of psychotherapy among transplanted patients are sparse. One study has shown both individual and group psychotherapy to be beneficial, with individual therapy leading to better improvement in comparison with group therapy (Abbey \& Farrow 1998).

\section{CONCLUSION}

Psychiatrists have multiple roles in the transplantation process. Initial psychiatric consultation addresses mental status and decision-making capacity. It is supplemented by standardized cognitive testing and psychosocial status assessment. In patients with a history of mental health issues, psychiatrists consider the risks of exacerbation or recurrence of psychiatric disorder. When deciding about psychotropic medication, psychiatrist must be aware of pharmacokinetic and pharmacodynamics issues due to organ failure and potential interactions involving immunosuppressant medications. Evaluation of emotional and cognitive preparedness for transplantation and therapy compliance is of the utmost importance. Consultation concludes with an overall assessment of patient's psychosocial strengths and limitations, giving summary of recommended interventions to optimize his or her candidacy for transplantation. In the process of donation, psychiatrists need to estimate psychological suitability and the motivation of the donor. Both from clinical practice and the scientific 
literature, it is known that psychiatric consultation has an important role in comprehensive candidate selection according to medical indications. However, due to different protocol schemes, transplantation teams still need to be reminded of psychological aspects and the role of psychiatrists as regular participants during the transplantation process. Psychiatric interventions are aimed to serve not only the needs of the patient and other transplant candidates, but also society in general, regarding optimal organ recruitment and helping recovery to optimal life quality and functioning.

\section{Acknowledgements:}

The authors would like to thank Gordon Johnson for his help and advice in preparing the article.

\section{Conflict of interest: None to declare.}

\section{Contribution of individual authors:}

Vesna Medved has been involved with conception and design of the paper, literature search, manuscript preparation, critically revising it and giving the final approval of the version to be submitted.

Sara Medved made substantial contribution to conception and design, literature search, has participated in drafting the article and revising it.

Milena Skočić Hanžek made substantial contribution to literature search and writing the article (especially regarding hearth transplantation), has participated in revising it.

\section{References}

1. Abbey S, Farrow S: Group therapy and organ transplantation. Ind J Group Psychother 1998; 48:163-85

2. Adam R, McMaster P, O'Grady JG, Castaing D, Klempnauer JL, Jamieson $N$ et al.: European Liver Transplant Association. Evolution of liver transplantation in Europe: report of the European Liver Transplant Registry. Liver Transpl 2003; 9:1231-1243

3. Akaho R, Sasaki T, Yoshino M, Hagiya K, Akiyama $H$ \& Sakamaki H: Bone marrow transplantation in subjects with mental disorders. Psychiatry Clin Neurosci 2003; 57:311-315

4. Anil Kumar BN \& Mattoo SK: Organ transplant \& the psychiatrist: an overview. Indian J Med Res 2015; 141:408-416

5. Barbour KA, Blumenthal JA \& Palmer SM: Psychosocial issues in the assessment and management of patients undergoing lung transplantation. Chest 2006; 129:1367-1374

6. Bianchi G, Marchesini G, Nicolino F, Graziani R, Sgarbi D, loquercio $C$ et al.: Psychological status and depression in patients with liver cirrhosis. Dig Liver Dis 2005; 37:593-600

7. Bouhlel S: Can patients with schizophrenia undergo renal transplantation with success? Saudi J Kidney Dis Transpl 2014; 25:605-9

8. Bunzel B \& Laederach-Hofmann K: Solid organ transplantation: are there predictors for posttransplant noncompliance? A literature overview. Transplantation 2000; 70:711-6
9. Burra P, Germani G, Gnoato F, Lazzaro S, Russo FP, Cillo $U$ et al.: Adherence in liver transplant recipients. Liver Transpl 201;17:760-70

10. Carney EF: The psychology of exraordinary altruism. Nat Rev Nephrol 2017; 13:383

11. Chenyu Y, Yamin Z, Ying Z,Yi l, Jianlin $J$ \& Hao C: Anxiety, depression and associated factors among inpatients waiting for heart transplantation. Shanghai Arch Psychiatry 2013; 25:165-173

12. Coffman KL, Hoffman A, Sher L, Rojter S, Vierling $J$ \& Makowka L: Treatment of the postoperative alcoholic liver transplant recipient with other addictions. Liver Transpl Surg 1997; 3:322-7

13. De Bleser L, Matteson M, Dobbels F, Russell C\& De Geest S: Interventions to improve medication-adherence after transplantation: a systematic review. Transpl Int 2009; 22:780-797

14. Dew MA, DiMartini AF, De Vito Dabbs A, Myaskovsky $L$, Steel $J \&$ Unruh $M$ et al.: Rates and risk factors for nonadherence to the medical regimen after adult solid organ transplantation. Transplantation 2007; 83:858873

15. Dew MA, Switzer GE, DiMartini AF, Matukaitis J, Fitzgerald $M G$ \& Kormos RL: Psychosocial assessments and outcomes in organ transplantation. Prog Transplant 2000; 10:239-59

16. Falkenstein K, Flynn L, Kirkpatrick B, Casa-Melley A \& Dunn S: Non-compliance in children post-liver transplant. Who are the culprits? Pediatr Transplant 2004; 8:233-6

17. Felnhofer A, Kothgassner OD \& Klier C: How to prevent depression? Current directions and future challenges in children with chronic medical conditions. Psychiatr Danub 2016; 28:441-451

18. Gilchrist NA, Asoh I \& Greenberg B: Atypical antipsychotics for the treatment of ICU delirium. $J$ Intensive Care Med 2012; 27:354-61

19. Hart A, Smith JM, Skeans MA, Gustafson SK, Stewart DE, Cherikh WS et al.: OPTN/SRTR 2015 Annual Data Report: Kidney. Am J Transplant 2017; 17:21-116

20. Hartmann A, Heilmann C, Kaps J, Bejesdorf F, Zah W, Albert $W$ et al.: Body image after heart transplantation compared to mechanical aortic valve insertion. Int $J$ Psychiatry Clin Pract 2017; 21:277-282

21. Inouye SK, Westendorp RG \& Saczynski JS: Delirium in elderly people. Lancet 2014; 383:911-22

22. Inspector Y, Kutz I \& David D: Another person's heart: magical and rational thinking in the psychological adaptation to heart transplantation. Isr J Psychiatry Relat Sci 2004; 41:161-73

23. Iruzubieta P, Crespo $J$ \& Fábrega E: Long-term survival after liver transplantation for alcoholic liver disease. World J Gastroenterol 2013; 19:9198-9208

24. Jowsey SG, Taylor KL, Schneekloth TD \& Clark MM: Psychosocial challenges in transplantation. J Psych Practice 2001;7:404-414

25. Kaba E, Thompson DR, Burnard P, Edwards D \& Theodosopoulou E: Somebody else's heart inside me: a descriptive study of psychological problems after a heart transplantation. Issues Ment Health Nurs 2005; 26:611-25

26. Khan A, Fabre LF \& Rudolph R: Venlafaxine in depressed outpatients. Psychopharmacol Bull 1991; 27:141-144

27. Mackie J, Groves K, Hoyle A, Garcia C, Garcia R, Gunson B et al.: Orthotopic liver transplantation for alcoholic liver disease: a retrospective analysis of 
survival, recidivism, and risk factors predisposing to recidivism. Liver Transpl 2001; 7:418-427

28. Mai I, Stoemer E, Bauer S, Kruger H, Budde $K$ \& Roots I: Impact of St. John's wort treatment on the pharmacokinetics of tacrolimus and mycophenolic acid in renal transplant patients. Nephrol Dial Transplant 2003; 18:819-822

29. McCallum $S$ \& Masterton G: Liver transplantation for alcoholic liver disease: a systematic review of psychosocial selection criteria. Alcohol Alcohol 2006; 41:358-63

30. Mi H, Li S, Zhao X, Li L \& Hu W: Reversible posterior white matter encephalopathy complicated with cerebral hemorrhage after orthotopic liver transplantation. Biomed Res 2017; 28:5972-5974

31. Milaniak I, Wilczek-Ruzyczka E, Wierzbicki K, Keidziora $A \&$ Przybylowski P: The effect of clinical variables on distress and depressive symptoms among heart transplant recipients. Heart Lung 2016; 48:1761-6

32. Montgomery SA: Safety of mirtazapine: a review. Int Clin Psychopharmacol 1995; 10(Suppl 4):37-45

33. Muscara F, Burke K, McCarthy MC, Anderson VA, Hearps SJC, Hearps SJ et al.: Parent distress reactions following a serious illness or injury in their child: a protocol paper for the take a breath cohort study. BMC Psychiatry 2015; 15:153

34. Naqui R: Evaluation of psychiatric issues in renal transplanting setting. Indian J Nephrol 2015; 25:321-325

35. Panocchia N, Bossola $M \&$ Vivanti G: Transplantation and mental retardation: what is the meaning of a discrimination? Am J Transplant 2010; 10:727-30

36. Parekh PI, Blumenthal JA, Babyak MA, Merrill K, Carney RM, Davis RD et al.: Psychiatric disorder and quality of life in patients awaiting lung trans-plantation. Chest 2003; 124:1682-1688

37. Parikh ND, Ladner D, Abecassis M \& Butt Z: Quality of life for donors after living donor liver transplantation: $A$ review of the literature. Liver Transpl 2010; 16:1352-1358

38. Robaeys G, Nevens F, Stärkel P, Colle I, Van Eyken P, Bruckers $L$ et al.: Previous intravenous substance use and outcome of liver transplantation in patients with chronic hepatitis C infection. Transplant Proc 2009; 41:589-594

39. Rogal SS, Landsittel D, Surman O, Chung RT \& Rutherford A: Pre-transplant depression, antidepressant use, and outcomes of orthotopic liver transplantation. Liver Transpl 2011; 17:251-260

40. Rovelli M, Palmeri D, Vossler E, Bartus S, Hull D \& Schweizer $R$ : Noncompliance in organ transplant recipients. Transplant Proc 1989; 21(1 pt 1):833-834

41. Sadala ML \& Stolf NA: Heart transplantation experiences: a phenomenological approach. J Clin Nurs 2008; 17:217-25

42. Saudi J: Kidney Dis Transpl 2014; 25:605-9

43. Schulz KH \& Kroencke S: Psychosocial challenges before and after organ transplantation. Transpl Res Risk Manag 2012; 7:45-58

44. Smith PJ, Blumenthal JA, Trulock EP, Freedland KE, Carney RM, Davis RD et al.: Psychosocial predictors of mortality following lung transplantation. Am Journal of Transplantation 2016; 16:271-277

45. Tandon P, Goodman KJ, Ma MM, Wong WW, Mason AL, Meeberg $G$ et al.: A shorter duration of pre-transplant abstinence predicts problem drinking after liver transplantation. Am J Gastroenterol 2009; 104:1700-1706

46. Taylor D, Paton C\& Kapur S: Use of psychotropies in special patient groups. In: The Mandsley prescribing guidelines, 10th ed. UK: MGB Books, 2010; 335-463

47. Teperman $L W$ : Impact of pretransplant hepatic encephalopathy on liver posttransplantation outcomes. Int $J$ Hepatol 2013; 952828

48. Tome $S$ \& Lucey MR: Timing of liver transplantation in alcoholic cirrhosis. J Hepatol 2003; 39:302-307

49. Vamos M: Organ transplantation and magical thinking. Aust N Z J Psychiatry 2010; 44:883-7

50. Yates WR, LaBrecque DR \& Pfab D: Personality disorder as a contraindication for liver transplantation in alcoholic cirrhosis. Psychosomatics 1998; 39:501-511

51. Zoellner T\& Maercker A: Posttraumatic growth in clinical psychology - A critical review and introduction of a two component model. Clin Psychol Rev 2006; 26:626-653

Correspondence:

Professor Vesna Medved, $M D, P h D$

Department of Psychiatry, Zagreb University Hospital Centre

Kišpatićeva 12, 10000 Zagreb, Croatia

E-mail:vmedved@net.hr 